\title{
Farmacopuntura: nivel de neuroleptoanalgesia en felinos utilizando xilacina-tramadol en acupunto yintang
}

\author{
González, R. ${ }^{\text {; }}$ Arguello, J. ${ }^{1}$; Britez, C. ; Viveros, R.E. ${ }^{2}$; Bazán, Y. ${ }^{2}$ \\ ${ }^{1}$ Cátedra Técn. Operat., ${ }^{2}$ Dep. Post Grado, Facultad de Ciencias Veterinarias, \\ Universidad Nacional de Asunción, San Lorenzo, Paraguay. \\ E-mail: rgonzalez@vet.una.py
}

\begin{abstract}
Resumen
González, R.; Arguello, J.; Britez, C.;Viveros, R.E.; Bazán, Y.: Farmacopuntura: nivel de neuroleptoanalgesia en felinos utilizando xilacina-tramadol en acupunto yintang. Rev. Vet. 31: 1, 8-13, 2020. El presente trabajo fue realizado con el objetivo de determinar el efecto del protocolo xilacina-tramadol en acupunto yintang versus administración intramuscular, sobre el nivel de neuroleptoanalgesia en felinos sometidos a orquiectomía. Para tal fin, fueron utilizados 20 felinos clínicamente sanos, mayores de 8 meses de edad, con serología negativa a virus inmunodeficiencia felina y virus leucemia felina, divididos en dos grupos. El grupo 1 recibió premedicación vía intramuscular con xilacina $1 \mathrm{mg} / \mathrm{kg}+$ tramadol $2 \mathrm{mg} / \mathrm{kg}$ y el grupo 2 recibió premedicación vía subcutánea en el acupunto yintang con xilacina $0,1 \mathrm{mg} /$ $\mathrm{kg}+$ tramadol $2 \mathrm{mg} / \mathrm{kg}$. Luego de 15 minutos los pacientes fueron valorados según la Escala de Otero para la evaluación de sedación y 15 minutos después se estimó la valoración post quirúrgica, según la escala multidimensional de la UNESP-Botucatu para valorar el dolor agudo post-operatorio. Los resultados obtenidos no arrojaron diferencias estadísticamente significativas $(p>0,05)$ entre los niveles de neuroleptoanalgesia registrados en ambos grupos en estudio.
\end{abstract}

Palabras clave: felinos, anestesia, neuroleptoanalgesia, acupunto yintang, xilacina, tramadol, orquiectomía.

\begin{abstract}
González, R.; Arguello, J.; Britez, C.; Viveros, R.E.; Bazán, Y.: Pharmacopuncture: level of neuroleptoanalgesy in felines using xylacine-tramadol in yintang acupunct. Rev. Vet. 31: 1, 8-13, 2020. The present work was carried out with the objective of determining the effect of the protocol xylacine-tramadol in acupunct yintang versus intramuscular administration on the level of neuroleptoanalgesy in felines subjected to orchiectomy. For this purpose, 20 felines apparently healthy, older than 8 months of age were used, with negative serology to virus feline immunedeficiency and virus feline leukemia, divided into two groups. The group 1 received pre-medication via intramuscular with xylacine $1 \mathrm{mg} / \mathrm{kg}+\operatorname{tramadol} 2 \mathrm{mg} / \mathrm{kg}$, and group 2 received pre-medication via acupunct yintang with xylacine $0,1 \mathrm{mg} / \mathrm{kg}+$ tramadol $2 \mathrm{mg} / \mathrm{kg}$. After 15 minutes, the patients were evaluated according to the Otero Scale for the evaluation of sedation, and the post-surgical evaluation was performed 15 minutes after the procedure, according to the multidisciplinary scale of the UNESP-Botucatu for the evaluation of the acute postoperative pain. The results obtained did not show statistically significant differences $(p>0,05)$ regarding the level of neuroleptoanalgesia between both study groups.
\end{abstract}

Key words: felines, anesthesia, neuroleptoanalgesy, yintang acupunct, xylacine, tramadol, orchiectomy.

\section{INTRODUCCIÓN}

El arte y la práctica de la anestesia se basan en un conocimiento general de los términos que describen los efectos de la misma en los animales, la farmacología de las sustancias anestésicas y de sus antagonistas, así como los métodos correctos de administración del fármaco anestésico. La anestesia es la pérdida total de

Recibido: marzo 2020 / Aceptado: junio 2020 las sensaciones en un área orgánica o en todo el organismo, inducida por un fármaco o fármacos que deprimen la actividad del tejido nervioso ya sea localmente (periférico) o general (central).

Es un proceso reversible, cuyo objetivo es producir un medio de inmovilización química cómodo, seguro, eficaz, además de barato, de forma que se puedan llevar a cabo los procedimientos clínicos con un mínimo de estrés, dolor, molestias y efectos secundarios tóxicos para el paciente y para el anestesista ${ }^{11}$. 
Los felinos domésticos presentan algunas características que son importantes para el tratamiento: sus métodos de sujeción y/o contención, las vías alternativas de administración de la preanestesia, la inducción, la recuperación anestésica, la excitación post anestésica y el tratamiento del dolor, entre otras.

Los gatos sintetizan los conjugados glucorónidos muy lentamente, porque son deficitarios en la enzima UTP-glucoronil-transferasa. Así, todas aquellas drogas o medicamentos que requieran de la glucoronotransformación hepática en su metabolismo, tendrán una vida media más prolongada. Sedantes, analgésicos y anestésicos -en su mayoría- necesitan de este proceso para su eliminación y por lo tanto, la recuperación anestésica será siempre más lenta en los felinos que, por ejemplo, en los caninos ${ }^{10}$.

Los agonistas $\alpha 2$-adrenérgicos se unen a receptores adrenérgicos $\alpha 2$ presinápticos en el $\mathrm{SNC}$ e inducen hiperpolarización e inhibición de la liberación de noradrenalina y dopamina. La activación de estos adrenoreceptores puede ejercer efectos analgésicos, sedantes, anticonvulsivantes, tranquilizantes y relajantes musculares.

Son sedantes y analgésicos potentes porque estimulan los receptores localizados en el SNC, pero tienen muchos efectos secundarios derivados de la activación de los receptores distribuidos por todo el organismo, provocando una hipertensión transitoria seguida por bradicardia, hipotensión y bloqueo atrio-ventricular. Además de encontrarse en el SNC, los receptores $\alpha 2$ se hallan en aparato digestivo, útero, riñones y plaquetas.

En anestesia veterinaria se utiliza xilacina, medetomidina y dexmedetomidina. Respecto a los agonistas adrenérgicos $\alpha 2$, la xilacina es el fármaco más antiguo de este grupo ${ }^{6}$. El tramadol es un analgésico considerado como opioide, actuando sobre las células nerviosas de la médula espinal y el cerebro, mostrando un agonismo, relativamente débil y no selectivo sobre los receptores opioides $m u$, delta y kappa; sin embargo, su efecto analgésico se debe en gran parte a su acción sobre los neurotransmisores, ya que facilita la liberación de serotonina e inhibe la recaptación de nor-epinefrina.

La dosis utilizada en felinos es de $2-4 \mathrm{mg} / \mathrm{kg}$ cada $12 \mathrm{~h}$ vía oral. En general es bien tolerada por los gatos, habiendo demostrado buena analgesia sin cambios significativos en los parámetros fisiológicos, aún con la dosis más alta recomendada $(4 \mathrm{mg} / \mathrm{kg})^{8,9}$.

$\mathrm{La}$ acupuntura representa una parte integral y muy importante de la medicina tradicional china, habiendo sido utilizada durante 2500 años. La técnica consiste en introducir agujas extremadamente finas en puntos específicos del cuerpo, denominados puntos de acupuntura, induciendo múltiples respuestas que actúan -entre otros- sobre el sistema nervioso.

La fármaco-puntura consiste en la aplicación de drogas en puntos específicos y este método combina la eficacia de la estimulación del acupunto con las propiedades farmacológicas de las drogas aplicadas, potenciando el estímulo mecánico in-situ y produciendo efectos similares a aquellos causados por el uso de esas drogas en terapias convencionales. La farmacopuntura utiliza subdosis de drogas.

En seres humanos y animales, la medicina tradicional china requiere una somera descripción del lugar donde se encuentra el punto yintang, en el cual se generará el efecto sedante. En perros se demostró que la inyección de $0,01 \mathrm{mg} / \mathrm{kg}$ de xilacina en el acupunto yintang reducía en un $32 \%$ la cantidad necesaria de tiopental para inducción de anestesia en perros. En la mayoría de casos, se observó que el uso de $\alpha$-agonistas en el acupunto yintang produjo efectos sedantes clínicamente relevantes con la ventaja de reducir los efectos secundarios asociados al uso de $\alpha 2$-agonistas en comparación con un grupo tratado con la dosis convencional de $0,1 \mathrm{mg} / \mathrm{kg}$ de xilacina ${ }^{17}$.

El objetivo del trabajo fue evaluar el efecto de la xilacina-tramadol en acupunto yintang y compararlo con la administración intramuscular de la misma combinación, en la neuroleptoanalgesia de felinos sometidos a orquiectomia de rutina.

\section{MATERIAL Y MÉTODOS}

El estudio experimental fue realizado en la Facultad de Ciencias Veterinarias de la Universidad Nacional de Asunción y las determinaciones serológicas fueron realizadas en el Laboratorio CEDIVEP, ubicado en la ciudad de San Lorenzo, Paraguay.

Fueron utilizados 20 animales de la especie felina, sin distinción de raza, mayores de 8 meses de edad, machos enteros, clínicamente sanos, sometidos a orquiectomía, ASA I o ASA II, negativos a virus de la inmunodeficiencia felina y de la leucemia felina. El tipo de muestreo fue probabilístico y aleatorio simple.

En la evaluación pre quirúrgica se determinaron los datos fisiológicos relativos a la temperatura, frecuencia respiratoria, frecuencia cardiaca, pulso y presión arterial sistólica, diastólica y media (Figura 1). Luego se realizó la sujeción semiológica de los pacientes, los cuales fueron divididos al azar en dos grupos, con igual cantidad de machos felinos en cada uno, asignándose un protocolo diferente a cada grupo.

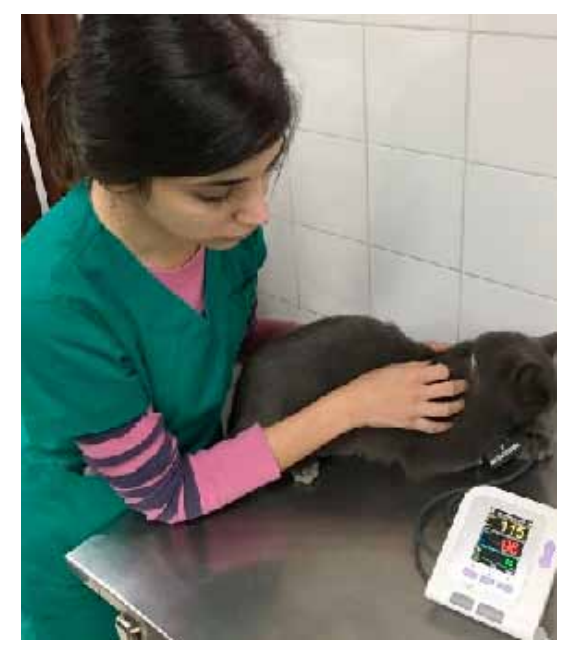

Figura 1. Medición de presión arterial. 


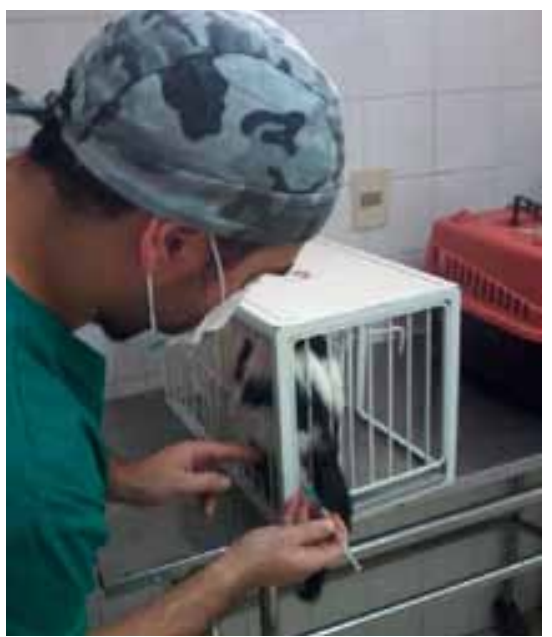

Figura 2. Administración intramuscular.

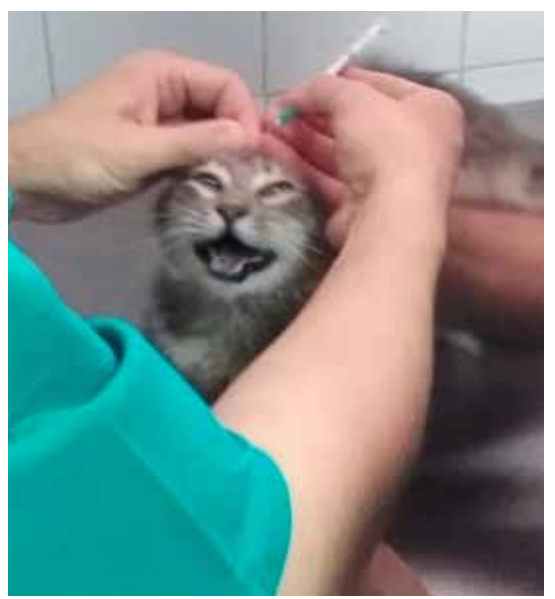

Figura 3. Administración en yintang.

Grupo 1: premedicación vía intramuscular con xilacina $(1 \mathrm{mg} / \mathrm{kg})+$ tramadol $(2 \mathrm{mg} / \mathrm{kg})$. Luego de aguardar 15 minutos se realizó la evaluación según una escala de valoración ${ }^{12}$.

Grupo 2: premedicación vía acupunto yintang con xilacina $(0,1 \mathrm{mg} / \mathrm{kg}+$ tramadol $2 \mathrm{mg} / \mathrm{kg})$. Se aguardaron 15 minutos, tras lo cual se efectuó la evaluación según la escala de valoración ya citada ${ }^{12}$.

Posteriormente, una vez evaluada la neuroleptoanalgesia pre-quirúrgica, se realizó la inducción con propofol IV (4-8 mg/kg), la intubación con traqueotubo $\mathrm{N}^{\circ} 2,5$ y el mantenimiento con isoflurano $(1,5-2,5 \%)$.

Una vez que el paciente se encontró en plano anestésico se procedió a la tricotomía de la región perineal, previa antisepsia con solución jabonosa de clorhexidina al $2 \%$, retirando el exceso con gasas embebidas con alcohol etílico de $96^{\circ}$, totalizando 3 lavados. Preparado el paciente para el procedimiento quirúrgico, se lo trasladó al quirófano, donde se procedió a la embrocación con solución de clorhexidina al $0,5 \%$.

Los pacientes fueron monitorizados utilizando un equipo multi-paramétrico de uso veterinario. Intra-quirúrgicamente fueron tenidos en cuenta los datos de las frecuencias cardíaca y respiratoria para la valoración del dolor, como así también la presión arterial sistólica, diastólica y media, medidas cada 5 minutos.

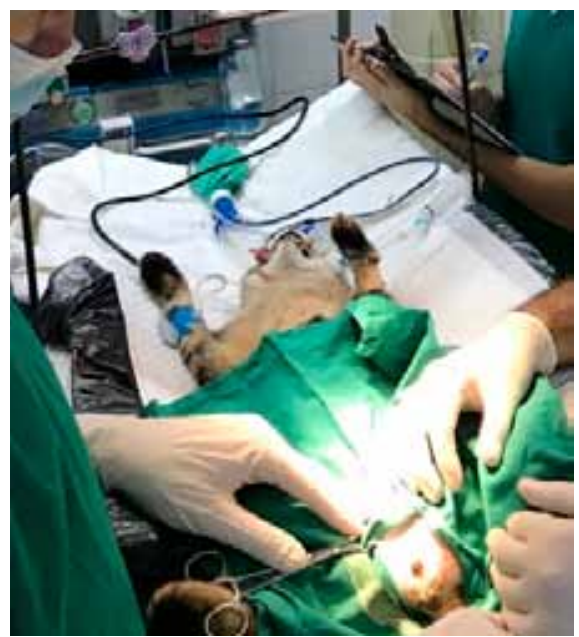

Figura 4. Realización del procedimiento y monitorización intraquirúrgica.

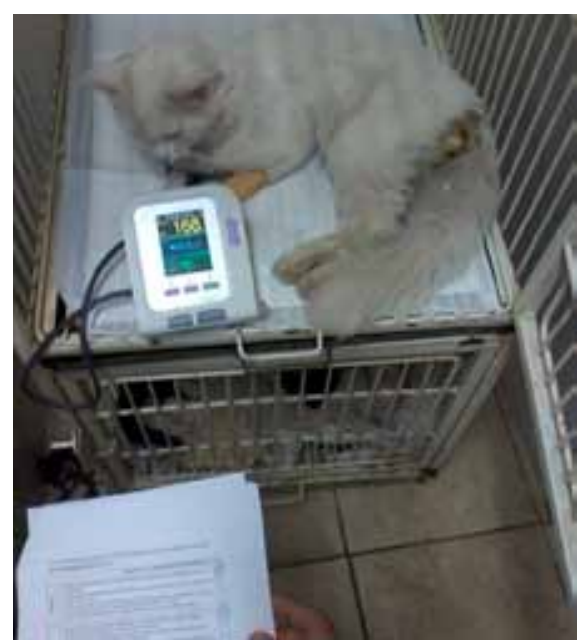

Figura 5. Evaluación post-quirúrgica utilizando la escala de UNESP-Botucatu.

Para el periodo post-quirúrgico fue utilizada la escala multidimensional de la UNESP-Botucatu para la evaluación del dolor agudo postoperatorio en gatos. Fueron tenidos en cuenta los datos de postura, comodidad, actividad, actitud, reacción a la palpación de la herida y vocalización, medidos al finalizar el procedimiento quirúrgico.

Los pacientes fueron medicados durante el período post-operatorio con amoxicilina de larga acción (22 $\mathrm{mg} / \mathrm{kg}$ IM cada $48 \mathrm{~h}, 2$ aplicaciones) y meloxicam a dosis de $0,3 \mathrm{mg} / \mathrm{kg}$ IM cada 24 h durante 3 días.

Los datos hematológicos fueron contrastados con los valores de referencia utilizados en el laboratorio para las series roja (eritrocitos, hematocrito, hemoglobina, volumen corpuscular medio y concentración media de hemoglobina) y blanca (neutrófilos, linfocitos, monocitos, eosinófilos y basófilos).

La neuroleptoanalgesia se evaluó por medio de métodos cuali y cuantitativos, observándose las actitudes y posturas, otorgándose valores ordinales clasificados en grados basados en el pre-quirúrgico, según escala propuesta para ponderar el grado de neuroleptoanalgesia en pequeños animales ${ }^{12}$. 
La evaluación post quirúrgica se realizó según la escala multidimensional de la UNESP-Botucatu para la estimación del dolor agudo postoperatorio en gatos ${ }^{3}$, teniendo en cuenta la postura, comodidad, actividad, actitud, reacción a la palpación de la herida y vocalización, valorados al finalizar el procedimiento quirúrgico. Para las variables cualitativas, se utilizó el chi cuadra$d o$, con un nivel de confianza del $95 \%$, obtenido a partir del paquete estadístico Infostat 2008.

Los parámetros evaluados en la neuroleptoanalgesia del pre-quirúrgico fueron: grado 0 (excitación, inquietud e hiperactividad ante estímulos), grado 1 (normal, despierto y completamente alerta), grado 2 (sedación leve, ambulación, decúbito esternal, adormecido pero fácil de despertar), grado 3 (sedación moderada, decúbito lateral, sin deambulación, adormecido, difícil de despertar) y grado 4 (sedación manifiesta, dormido, no despierta ante estímulos verbales o táctiles) ${ }^{12}$.

Los diferentes parámetros se evaluaron según el margen de referencia para la especie: frecuencia respiratoria (20-40 resp/min), frecuencia cardíaca (120-240 lat/min), oximetría de pulso $\left(\mathrm{SpO}_{2}\right.$ sobre $\left.95 \%\right)$ y presión arterial sistólica (100-160 $\mathrm{mmHg}$ ), diastólica (60-100 $\mathrm{mmHg}$ ) y media $(80-110 \mathrm{mmHg})$. El efecto neuroleptoanalgésico se consideró adecuado cuando estos datos encuadraron en los márgenes de referencia para la especie $^{15}$.

La interpretación de la neuroleptoanalgesia postquirúrgica se realizó según la escala multidimensional de la UNESP-Botucatu para la evaluación del dolor agudo postoperatorio en gatos, y fue interpretada según los valores obtenidos para un total de $0-30$, siendo $0-8=$ dolor leve, $9-20=$ dolor moderado y $21-30=$ dolor intenso ${ }^{3}$.

\section{RESULTADOS Y DISCUSIÓN}

Tabla 1. Escala de evaluación de sedación de los pacientes.

\begin{tabular}{cccc}
\hline grados & vía intramuscular & acupunto yintang & total \\
\hline 1 & 4 & 4 & 8 \\
2 & 5 & 4 & 9 \\
3 & 1 & 2 & 3 \\
total & 10 & 10 & 20 \\
\hline
\end{tabular}

En la Tabla 1 se observan los valores obtenidos luego de la administración de las drogas (sedación) ${ }^{12}$. En el grupo 1 (acupunto yintang), del total de 10 felinos utilizados, 4 obtuvieron un valor grado 1 (normal, despierto y completamente alerta), y otros 4 obtuvieron un valor grado 2 (sedación leve, decúbito esternal, adormecimiento con fácil despertar y capacidad de deambular). Dos de ellos obtuvieron un grado 3 (sedación moderada, decúbito lateral, adormecimiento con difícil despertar e incapacidad para la deambulación). Ninguno de ellos obtuvo los grados 0 ni 4.

En el grupo 2 (vía intramuscular), del total de 10 felinos, 4 de ellos obtuvieron un valor grado 1 (normal, despierto y completamente alerta), 5 obtuvieron valor grado 2 (sedación leve, decúbito esternal; adormecimiento con fácil despertar, capacidad para deambular). Uno de ellos obtuvo un grado 3 (sedación moderada, decúbito lateral, adormecimiento con difícil despertar e incapacidad para deambular). Ninguno de ellos obtuvo los grados 0 ni 4.

El elevado número de felinos con sedación grado 1, o sea despiertos y completamente alertas, puede estar relacionado con el alto nivel de excitación previo a la administración de las drogas, ya que se trata de especies fácilmente irritables, sumado al hecho de que se encontraban en un ambiente extraño, con personas a las cuales no estaban familiarizados. El nivel de sedación puede ser inferior al esperado cuando existe previamente estrés, miedo, excitación o dolor en el animal.

Como es bien sabido, el gato y los felinos en general, son animales que se estresan considerablemente cuando se los aparta de su territorio habitual o son sometidos a variaciones en su ambiente. El profesional debe estar familiarizado con una serie de manifestaciones que le indiquen el nivel de estrés del animal, de forma que pueda prevenir una respuesta agresiva o exacerbada ${ }^{6}$.

Otro factor a tener en cuenta son los sitios de aplicación de la droga. En la administración intramuscular o subcutánea, el comienzo de la acción puede tardar de 10 a 15 minutos en aparecer. Es posible que el efecto máximo no se consiga durante muchos minutos o incluso horas, dependiendo del riego sanguíneo de los tejidos en el punto de inyección, de la absorción del fármaco y del ritmo metabólico. La duración de la acción es más prolongada que la vía intravenosa y si se administra por vía transdérmica, el máximo efecto sistémico puede no aparecer hasta después de muchas horas ${ }^{11}$.

Se debe tener en cuenta que el objetivo de la premedicación anestésica es ayudar a la inmovilización del animal mediante la modificación de su conducta, para que facilite el trabajo con el paciente, reduzca el estrés, prevenga o limite el dolor antes, durante y después de la cirugía, produzca relajación muscular, disminuya la cantidad de fármacos potencialmente peligrosos, promueva la inducción, mantenimiento y recuperación de la anestesia, evite complicaciones, prevenga los efectos adversos y potencialmente tóxicos de los fármacos administrados y reduzca al mínimo la actividad refleja vegetativa, ya sea de origen simpático o parasimpático ${ }^{11}$.

La xilacina se absorbe rápidamente después de la inyección intramuscular, por lo cual la concentración plasmática máxima ocurre después de 15 a 25 minutos. Por otro lado, una décima parte de la dosis de xilacina en el acupunto yintang induce una normal a media sedación, con el efecto sedante observado a los 30 minutos después de la inyección. El inicio es lento, debido a que la administración en el punto de acupuntura da como resultado una absorción subcutánea ${ }^{5}$.

En la mayoría de los pacientes (19 de 20), fue observado un efecto émetico luego de la administración intramuscular de xilacina-tramadol, así como también en la vía acupunto yintang. Los alfa-agonistas típica- 
mente inducen el vómito mediante la estimulación de la zona de activación de los quimiorreceptores, la xilacina provoca emesis durante la sedación del $50 \%$ de los perros y el $90 \%$ de los gatos ${ }^{5}$. En algunos pacientes fue observado además cierto grado de hemorragia en la inyección acupunto.

Los datos obtenidos de la escala de evaluación de sedación, fueron sometidos a análisis estadísticos, utilizando la prueba de Chi cuadrado, con un $95 \%$ de confianza, no encontrándose diferencias estadísticamente significativas entre ambos grupos de estudio $(p>0,05)$. Al término de los efectos anestésicos, se realizó la evaluación de la intensidad de dolor agudo post-operatorio mediante la escala multidimensional de la UNESP-Botucatu, realizando la calificación cuando el paciente se encontraba completamente despierto.

Tabla 2. Escala multidimensional de la UNESP-Botucau para la evaluación del dolor agudo posoperatorio.

\begin{tabular}{lcc}
\hline \multirow{2}{*}{ administración } & \multicolumn{2}{c}{ escala (score total: 30 puntos) } \\
\cline { 2 - 3 } acupunto yintang & con dolor & sin dolor \\
vía intramuscular & 0 animales & 10 animales \\
total & 0 animales & 10 animales \\
\hline
\end{tabular}

Como se puede observar en la Tabla 2, según la escala multidimensional de la UNESP-Botucatu para la evaluación del dolor agudo post-operatorio, en el grupo de acupunto yingtang, 8 felinos presentaron un valor menor a 8 (siendo $0-8=$ dolor leve), y 2 (dos) felinos con valores mayores a 8 pero menores a $20(9-20=$ dolor moderado), no presentándose ningún felino con dolor intenso $(21-30=$ dolor intenso).

En el grupo de administración intramuscular, 7 felinos presentaron un valor menor a 8 (siendo $0-8=$ dolor leve) y 3 felinos ostentaron valores mayores a 8 pero menores a $20(9-20=$ dolor moderado), no presentándose ningún felino con dolor intenso $(21-30=$ dolor intenso).

Los alfa-2 agonistas son excelentes sedantes; producen una sedación dosis-dependiente y proporcionan una analgesia similar a la observada por la activación de los receptores opioides. La sedación se produce como consecuencia de un descenso en la liberación de nor-epinefrina y la analgesia, al estimular receptores que intervienen en la nocicepción a nivel del cerebro y la medula espinal ${ }^{13}$.

Existen dos razones principales por las cuales estos compuestos presentan efectos similares a los opioides. En primer lugar, los receptores alfa-2 adrenérgicos y $\mu$-opioides se encuentran en regiones similares del cerebro, incluso en las mismas neuronas. La segunda razón es que cuando se unen a sus receptores, los fármacos alfa-2-adrenérgicos y los opioides dan lugar a la activación del sistema de transducción (proteína G). Como consecuencia, se produce la apertura de canales de $\mathrm{K}+$ en la membrana neural, lo cual da lugar a la perdida de $\mathrm{K}+$ intracelular y modifica la carga eléctrica, de forma que la célula no responde a los estímulos excitatorios. Existen evidencias a nivel experimental y clínico, que demuestran que la analgesia obtenida con los agonistas alfa-2 dura la mitad de tiempo que la acción sedante y que no es efectiva en todos los tipos de dolor ${ }^{16}$.

La duración del efecto de la xilacina depende de la dosis administrada, siendo de 1,5 horas la persistencia promedio tras la administración IM o SC (vías de elección en pequeños animales). El inicio de la acción se retrasa 10-15 minutos y el efecto analgésico tiene una duración de sólo 15-30 minutos, aún cuando el efecto sedante se mantiene durante 1 ó 2 horas ${ }^{2}$.

El tramadol es un analgésico opioide que actúa sobre el sistema nervioso central. Es un agonista puro no selectivo de los receptores opioides $\mu, d, k$ con una mayor afinidad por los receptores $\mu$. Además, in vitro inhibe la re-captación de noradrenalina y serotonina. Estos mecanismos pueden contribuir al efecto analgésico del tramadol. Se considera que la potencia de este último es de $1 / 10$ a $1 / 6$ la potencia de la morfina ${ }^{1}$.

El tramadol es interesante como analgésico postoperatorio y tiene la característica de actuar como opiáceo agonista puro, y además por bloqueo monoaminérgico ${ }^{4}$. Comparada con la morfina, luego de la administración parenteral su potencia analgésica es del $10 \%$ de la potencia de la morfina. El tramadol provee un alivio del dolor comparable con los péptidos opioides y su analgesia puede favorecerse con la combinación de un analgésico no opioide ${ }^{7,14}$.

Los datos obtenidos de la escala multidimensional de la UNESP-Botucau para la evaluación del dolor agudo post-operatorio, fueron sometidos a análisis estadísticos, utilizando la prueba de Chi cuadrado con un $95 \%$ de confianza, no encontrándose diferencias estadísticamente significativas entre ambos grupos de estudio $(\mathrm{p}>0,05)$.

En conclusión, ambos grupos obtuvieron igual cantidad de animales con sedación en grado 1 (4/4). Para el grado 2, cuatro felinos del grupo 1 (acupunto yintang) y cinco felinos del grupo 2 (intramuscular) lo presentaron. En cuanto a los grados 0,3 y 4, ningún felino los manifestó. Con respecto al nivel de analgesia posterior a la cirugía, ningún grupo manifestó dolor. Para futuras investigaciones se recomienda utilizar mayor número de animales y evaluar ambos protocolos en caninos, e incluso otras especies, además de utilizar distintos procedimientos quirúrgicos, demostrando así el uso cada vez más difundido de la farmacopuntura en la medicina veterinaria.

Agradecimientos. Por la colaboración prestada, a E. Maldonado (Dirección Académica, Facultad de Ciencias Veterinarias, Universidad Nacional de Asunción).

\section{REFERENCIAS}

1. Argueta LR. 2015. Tramadol en asociación farmacológica en el control del dolor posoperatorio en protocolos 
analgésicos polimodales preventivos en anestesiología de equinos, pequeña especies y otras especies no convencionales. Rev Electr Vet Méx 16: 1-17.

2. Botana L. 2002. Farmacología y terapéutica veterinaria, McGraw-Hill, New York, p. 734.

3. Brondani JT, Crosignani N. 2014. Validez y confiabilidad de la versión en español de la escala multi-dimensional de la UNESP-Botucatu para evaluar el post-operatorio en gatos. Arch Med Vet 46: 477-486.

4. Cabezas MA, Sández I. 2007. Analgesia en gatos. Rev Asoc Madr Vet Anim Comp (España), 24: 4-10.

5. Cassu R et al. 2014. Efecto sedativo y clínico de la farmacopuntura con xilacina en perros. Acta Cirúrg Bras 29: 47-52.

6. Colmenero C, Calzadilla I. 2010. Singularidades anestésicas de los felinos. Panoram Act Med (España) 34: 922-928.

7. Da Silva L. 2015. Usos del tramadol. Rev Méd Peq Anim (Uruguay) 2: 1-28.

8. García CE. 2014. Farmacopuntura con tramadol como analgesia postoperatoria preventiva en la herniorrafia inguinal. Rev Cub Med Milit 43: 1-9.

9. Marin HJ. 2015. Farmacología práctica en gatos, Celsa Impr., México, p. 675.
10. Minovich FG, Paludi A. 2011. Medicina felina práctica, Ed. Multimédica, Barcelona, 648 p.

11. Muir WW, Hubell JA. 2008. Manual de anestesia veterinaria, $4^{\mathrm{a}}$ ed., Elsevier, Madrid, 643 p.

12. Otero, P. 2012. Dolor, evaluación y tratamiento en pequeños animales, Inter-Médica, Buenos Aires, $330 \mathrm{p}$.

13. Paddleford RR, Harvey RC. 1999. Alpha-2 agonists and antagonists. Vet Clin North Am (Small Anim Pract) 29: 737-745.

14. Pérez JD, Abejón JR. 2005. El dolor y su tratamiento a través de la historia. Rev Soc Españ Dolor 12: 373-384.

15. Radostits O. 1999. Medicina veterinaria, Mc.Graw Hill, Madrid, $2215 \mathrm{p}$.

16. Sinclair D. 2003. A review of the physiological effects of alfa 2-agonists related to the clinical use of medetomidine in small animal practice. Canad Vet J 44: 203-214.

17. Souza TM. 2015. Efecto sedativo de la microdosis de acepromazina en acupunto yintang en perros. Tesis $\mathrm{Mg} \mathrm{Sc}$. Univ Lusóf Humanid e Tecnol, Faculd Med Vet, Lisboa, Portugal, $69 \mathrm{p}$. 